\title{
Women Doctors Don't Get the Credit They Deserve
}

\author{
Debra L. Roter, DrPH' and Judith A. Hall, PhD ${ }^{2}$ \\ 'Department of Health, Behavior and Society, Johns Hopkins Bloomberg School of Public Health, Baltimore, MD, USA; ${ }^{2}$ Department of Psychology, \\ Northeastern University, Boston, MA, USA.
}

$\mathrm{J}$ Gen Intern Med 30(3):273-4

DOI: $10.1007 / \mathrm{s} 11606-014-3081-9$

() Society of General Internal Medicine 2014

I n some very important respects, women are better doctors than men and they were better even before going to medical school. We are referring to skills that comprise the practice of patient-centered medicine, and we say this with all due qualification and acknowledgment of the enormous variation within and substantial overlap in these skills across gender. ${ }^{1}$ The explanation for women doctors' greater patientcenteredness is not based on "innate" gender differences, but on the societal norms and expectations that produce perceptibly different behavior repertoires that manifest in how women and men practice the art of doctoring. Unfortunately, along with this difference comes a documented gender bias when patients evaluate clinical performance.

Medicine has been transformed over the past 30 years by the confluence of two factors related to women doctoring: the dramatic increase in the number of women in the medical workforce and recognition of patient-centered medical practice as a cornerstone of care quality. The proportion of women entering medical school in the US rose from $10 \%$ in the $1970 \mathrm{~s}$ to $35 \%$ by the mid- 1980 s to just about $50 \%$ in $2000 ; 60 \%$ of the current medical school class at Johns Hopkins is female. While one-third of all practicing physicians in the US are women, they comprise $46 \%$ of physicians under the age of 35. The last several decades have also witnessed broad endorsement of patient-centeredness, which has come to signify the facilitation of active patient engagement in care through meaningful inclusion of patient perspectives and preferences. It also connotes an emotional connection such that the patient doesn't just feel cared for, but also cared about. Notably, the Institute of Medicine included patient-centeredness as key to shaping the nation's future agenda for the quality of health care, and the recently passed Patient Protection and Affordable Care Act is replete with references to patient-centered indicators of care quality.

Decades of research in nonclinical populations show that women in general are more relationship-oriented and egalitarian in their attitudes and conversational style, more expert in "reading" others' personality and emotions, more skilled in nonverbal communication, and higher scoring on measures of

Published online November 1, 2014 emotional intelligence compared to men. In short, women have a predilection to be patient-centered when they become doctors, and meta-analysis confirms that women doctors do indeed communicate with their patients differently than their male counterparts. ${ }^{1}$ Women doctors spend approximately $10 \%$ more time in a patient visit, and their patients (both male and female) talk more when with them and disclose more information about their medical symptoms, lifestyle, and emotional concerns. Women doctors communicate more positively, both verbally and nonverbally, they are more emotionally responsive (by talking more about emotions, and by expressing empathy and concern), and they are more likely to ask about and provide counseling in the areas of lifestyle and mental health. Moreover, there is no evidence that the gender differences apparent in psychosocial and socioemotional exchange are seen in biomedically focused discussions; male and female physicians discuss diagnosis, prognosis, and medical treatment quite similarly.

Although the magnitude of the effects for any given communication element is small, meaning there is substantial overlap across gender, the effects are significant and comparable to those of many well-established medical, psychological, behavioral, and educational interventions. The pattern of results is almost entirely consistent with the nonmedically related literature regarding gender differences in communication; the bottom line is that women doctors are more patientcentered than their male colleagues and they communicate like women.

But here is the rub: research suggests that patients don't give women doctors the credit they deserve. A meta-analysis of patient satisfaction and physician gender found, contrary to logical expectation and despite the general correlation between patient satisfaction and physicians' patient-centered behaviors, that the satisfaction difference between male and female physicians is extremely small, in fact almost nonexistent. ${ }^{2}$

Moreover, several recent studies in which patientcenteredness was experimentally manipulated or independently scored by trained coders show that patient-centered behavior is evaluated differently in male doctors than in women doctors. One of these studies, conducted with over 70 doctors and nearly 500 patients who had been treated by them, found a stronger positive correlation for male physicians between patient satisfaction and patient-centeredness measured by independent videotape coders than for female physicians. ${ }^{3}$ In 
another study, analogue patients (subjects who were asked to watch interactions while imagining themselves as the patient) rated the competence of male and female medical students conducting an objective structured clinical examination (OSCE) and showed the same gender bias - more patientcentered male students were rated as higher in competence than less patient-centered male students, while for female medical students there was no relation. ${ }^{4}$

And, a similar result was found in yet another study. In this instance, physicians' patient-centeredness was experimentally manipulated by using video recordings of actors playing the role of a physician who spoke to an unseen patient in a high or low patient-centered way, using identical scripts in each condition. Analogue patients then rated their satisfaction. Satisfaction was much more strongly predicted by patientcenteredness in male physicians than in female physicians. ${ }^{5}$ The experimental nature of the study assured that the differential effect in ratings of satisfaction was not due to any uncontrolled differences in how the male versus female physician-actors behaved, and can be logically attributed to gender bias in evaluating the physicians.

We can conclude that patients are less likely to attribute patient-centered behaviors performed by women doctors to clinical competence, and that this attribution error diminishes patients' satisfaction with these doctors. We believe that this is very likely the result of implicit or unconscious bias. Patients appreciate women doctors' patient-centeredness, but because of the overlap with the repertoire they expect from women, they do not see it as a marker of professional competence, but as expected female behavior, with the result that these skills are underappreciated when patients evaluate women doctors. Consequently, a patient-centered male doctor is seen as a good doctor, while a patient-centered female doctor is seen merely as a good woman.

The education of patients to more fully appreciate the value of patient-centered skills, no matter what their physician's gender, is an important public health goal that we believe is tied to how doctors themselves value these skills. We do not know if the subtle bias we have described in patients is also present in medical educators, preceptors and evaluators. We speculate that it is and think it is an important question to pursue. The bias could similarly be present among female physicians themselves who may undervalue their own patient-centered skills, at least in part because of the way it is perceived by those around them.

We believe change is coming to medicine, but it is unclear how long it will take to overcome biases that fail to equally credit women for their expertise. The days of physicians chanting under their breath, "Macho, macho doc, I wanna be a macho doc," as described by Perri Klass in A Not Entirely Benign Procedure: Four Years as a Medical Student (page $80)$, may be ending. However, we fear that for some years to come, women doctors may be chanting Aretha Franklin's refrain, along the lines of "All I'm asking is for a little respect."

Corresponding Author: Debra L. Roter, DrPH; Department of Health, Behavior and SocietyJohns Hopkins Bloomberg School of Public Health, Baltimore, MD 21205, USA (e-mail: droter1@jhu.edu).

\section{REFERENCES}

1. Roter DL, Hall JA. Physician gender and patient-centered communication: a critical review of empirical research. Annu Rev Public Health. 2004;25:497-519

2. Hall JA, Blanch-Hartigan D, Roter DL. Patients' satisfaction with male versus female physicians: a meta-analysis. Med Care. 2011;49:611-617.

3. Hall JA, Gulbrandsen P, Dahl FA. Physician gender, physician patientcentered behavior, and patient satisfaction: a study in three practice settings within a hospital. Patient Educ Couns. 2014;95:313-318.

4. Blanch-Hartigan D, Hall JA, Roter DL, Frankel RM. Gender bias in patients' perceptions of patient-centered behaviors. Patient Educ Couns. 2010;80:315-320.

5. Hall JA, Roter DL, Blanch-Hartigan D, Schmid Mast M, Pitegoff CA. How patient-centered do female physicians need to be? analogue patients' satisfaction with male and female physicians' identical behaviors. Health Commun. 2014;30:1-7. 